\title{
Frustración y conductas sociales
}

\author{
Frustration and Social Behavior \\ frustração e condutas sociais
}

\author{
Alba Elisabeth Mustaca* \\ Universidad Abierta Interamericana
}

Doi: http://dx.doi.org/10.12804/revistas.urosario.edu.co/apl/a.4643

\section{Resumen}

\begin{abstract}
A lo largo de la vida ocurren hechos que no son tal como lo esperábamos: algunas veces son mejores, otras peores; las neurociencias, incluida la psicología, estudia ampliamente los mecanismos y efectos que ellos provocan. Desde el punto de vista teórico, la frustración se define como la respuesta del organismo desencadenada cuando un sujeto experimenta una omisión o devaluación sorpresiva en la calidad o cantidad de un reforzador apetitivo, en presencia de señales previamente asociadas con un reforzador de mayor magnitud (Amsel, 1992). Este artículo se limitará a presentar las teorías principales sobre la frustración y ejemplos de las relaciones que existen entre ella y las respuestas sociales, en modelos animales y humanos.
\end{abstract}

Palabras clave: frustración, respuestas agonísticas, exclusión social, modelos animales y humanos.

\section{fibstract}

Throughout life, events play out in unexpected ways: sometimes they are better than expected and sometimes they are worse. The neurosciences, including psychology, widely study these mechanisms and their effects.
From a theoretical point of view, frustration is defined as the body response that is triggered when a subject experiences a surprising omission or devaluation in the quality or quantity of an appetitive reinforcer in the presence of signals previously associated with a reinforcer of greater magnitude (Amsel, 1992). In this article, I will present only the main theories of frustration and examples of the relationship between it and social responses in animal and human models.

Keywords: Frustration, agonistic responses, social exclusion, animal and human models.

\section{Resumo}

Ao longo da vida ocorrem fatos que não são tal como o esperávamos: algumas vezes são melhores, outras piores; as neurociências, incluída a psicologia, estuda amplamente os mecanismos e efeitos que eles provocam. Desde o ponto de vista teórico, a frustração define-se como a resposta do organismo desencadeada quando um sujeito experimenta uma omissão ou desvalorização supressiva na qualidade ou quantidade de um reforçador apetitivo, em presença de sinais previamente associadas a um reforçador de maior magnitude (Amsel, 1992). Neste artigo me limitarei a apresentar

* Centro de Altos Estudios de Ciencias Humanas y de la Salud (CAECIHS), Facultad de Psicología y Ciencias Humanas, Universidad Abierta Interamericana. Correspondencia. Correo electrónico: albamustaca@gmail.com

Cómo citar este artículo: Mustaca, A. E. (2018). Frustración y conductas sociales. Avances en Psicología Latinoamericana, 36(1), 65-81. doi: http://dx.doi.org/10.12804/revistas.urosario.edu.co/apl/a.4643 
as teorias principais sobre a frustração e exemplos das relações que existem entre ela e as respostas sociais, em modelos animais e humanos.

Palavras-chave: frustração, respostas agonísticas, exclusão social, modelos animais e humanos.

Una persona que busca un trabajo esperando un sueldo de $\$ 10000$ queda decepcionada si le ofrecen $\$ 5000$; otra, en cambio, si esperaba $\$ 3000$, puede sentirse eufórica con el mismo ofrecimiento; y un tercero, que esperaba $\$ 5000$, quedaría simplemente conforme. Con este ejemplo, se evidencia que las reacciones ante los hechos dependen, entre otros factores, de dos cuestiones: el valor del suceso en sí mismo, que es el tangible o absoluto (en el ejemplo, \$5000) y otro relativo o subjetivo, que es aquel que se espera recibir o tener (expectativa). La comparación entre lo esperado y lo recibido puede desencadenar respuestas emocionales, cognitivas y neurobiológicas de distinta índole: si se recibe más de lo esperado, se estará contento, eufórico y alegre; si es menor, se estará frustrado, enojado o triste; si es igual, se seguirá tranquilo y, se diría, "es lo que esperaba". En este artículo se hará referencia a las respuestas de los sujetos ante situaciones en las cuales reciben eventos más negativos a los esperados, que se conocen como frustración o violación de expectativas positivas. La intensidad y duración de las reacciones a estos eventos depende, entre otros factores, de la discrepancia existente entre lo que se espera (por historias de aprendizajes previos, diferencias individuales, etc.), y lo que se recibe. Las decepciones, si son muy intensas, pueden dejar profundas huellas en la conducta e inclusive en la salud, o fortalecer a la persona por contar con herramientas y capacidades para enfrentarlas.

Las situaciones que provocan estrés en la vida diaria contienen, en la mayoría de los casos, elementos que incluyen violación de expectativas positivas, como pérdidas, totales o parciales, de incentivos o reforzadores apetitivos, y no de estímulos aversivos que implican dolor proveniente de estímulos sensoriales, como una golpiza entre rivales. Esto se ve reflejado en numerosas investigaciones. La escala de valoración de reajuste social (SRRS, sigla en inglés) es uno de los instrumentos de medición más citados en la bibliografía sobre el estrés desde hace más de 50 años. Los SRRS constan de 43 ítems identificados a partir de experiencias clínicas psicológicas que requieren un reajuste emocional o que deben tratarse para prevenir efectos sobre la salud (Holmes \& Rahe, 1967). Esta escala, al igual que Scully, Tosi y Banning (2000), determina que los 5 principales eventos que una amplia población evaluó como estresantes fueron la pérdida de un ser querido, divorcio, separación, encarcelamiento y muerte de un familiar cercano. Las investigaciones clínicas muestran que la pérdida de un ser querido, del trabajo o problemas económicos pueden traer como consecuencia alteraciones en el área social, como mayor irritabilidad, depresión, agresividad o tendencia a volver a conductas no adaptativas del pasado, como alcoholismo o drogadicción (Bartrop, Luckhurst, Lazarus, Kiloh \& Peny, 1977; Hall \& Irwin, 2001; Rando, 1993; Stein \& Trestman, 1990). También suelen provocar una disminución inmunológica transitoria considerable, por lo cual la persona está más vulnerable y puede aumentar la probabilidad de adquirir enfermedades infecciosas, o para que se activen otras de origen genético (Kielcolt-Glaser et al., 1988; Mustaca, 2001; Mustaca \& Bentosela, 1995). La pérdida de reforzadores económicos también puede provocar serias alteraciones. Liprandi et al. (2012), por ejemplo, mostraron una estrecha relación temporal entre el aumento relativo de la mortalidad cardiovascular y el descenso del Producto Bruto Interno (PBI) en Argentina. Estas investigaciones correlacionales y clínicas son sugerentes, pero sus resultados no pueden determinar relaciones de causalidad. La muerte de un ser querido o la falta de trabajo, por ejemplo, pueden desencadenar estados o hábitos que en sí mismos provocan alteraciones psicofisiológicas, 
como no alimentarse bien, padecer insomnio, fumar mucho o estar distraídos y tener accidentes. En ese sentido, los diseños experimentales con humanos, manteniendo los códigos éticos, y los realizados con animales no humanos, como ratas o ratones, proveen más información básica sobre los efectos y mecanismos de la pérdida total o parcial de incentivos, ya que poseen un mayor control experimental, aunque no pueden explicar completamente la complejidad de la conducta humana. Es la interacción entre las investigaciones con humanos y con otros animales lo que traerá una mayor comprensión de las relaciones causales del comportamiento complejo y, por consecuencia, si esas conductas son perjudiciales, hallar formas de tratarlas o prevenirlas.

Los estudios sobre frustración comenzaron alrededor de 1930 (Tinklepaugh, 1928) y actualmente siguen siendo foco de interés. En sus comienzos, los investigadores buscaban realizar preparaciones de laboratorio que mostraran evidencia de algunas hipótesis, tales como que la frustración genera agresión, fijación, regresión y conflicto (Yates, 1975). Esta tendencia sigue existiendo, con el agregado de investigaciones neurobiológicas que desentrañan las bases neurales de la frustración.

La frustración es una palabra muy usada en la vida cotidiana. En psicología tiene al menos dos acepciones (Berkowitz, 1989). Dollard, Doob, Miller, Mowrer y Sears (1939) la consideraron como un estímulo (una interferencia) entre una secuencia de respuestas que se dirigen hacia una meta. Amsel (1958, 1992), en cambio, la define en función de las repuestas que tiene el organismo ante estímulos que implican un decremento, omisión o interferencias de reforzadores y desarrolla una teoría de la frustración, fundada en mecanismos asociativos. En este artículo se tendrá en cuenta el concepto de Amsel. La frustración o efectos paradójicos del reforzamiento (Amsel, 1992), contraste negativo o relatividad de los refuerzos (Flaherty, 1996) u omisión sorpresiva de reforzadores (Papini, 1997, 2003) se define en ese contexto como la respuesta del organismo (conductual, fisiológica y neural) desencadenada cuando un sujeto experimenta una devaluación sorpresiva en la calidad o cantidad de un reforzador apetitivo, en presencia de señales contextuales o temporales previamente asociadas con un reforzador de mayor magnitud. Los procedimientos para estudiarlos son variados, aunque todos ellos tienen en común la violación de una expectativa positiva que se adquirió mediante la historia de aprendizajes previos. En los modelos animales, la omisión de un estímulo apetitivo puede involucrar la eliminación total del reforzador apetitivo (extinción), la reducción parcial de su magnitud o calidad (contraste cuantitativo y cualitativo), la reducción en la tasa del reforzamiento (contraste conductual), un cambio de un reforzador preferido a otro menos preferido (contraste anticipatorio), o la interpolación de una barrera entre el sujeto y el reforzador cuando antes no lo estaba. Para evaluar los efectos de la disminución o ausencia de reforzadores sociales, se aíslan a los sujetos, en la mayoría de los casos en etapas de la infancia o adolescencia y se miden parámetros conductuales y psicofisiológicos en la adultez. Los instrumentos utilizados, en general, son los mismos que se usan para el estudio del aprendizaje: corredores lineales, laberintos, cajas de condicionamiento, campo abierto, privación social, entre otros no tan convencionales. Como incentivos se utilizan en la mayoría de los casos alimentos sólidos o líquidos de distinta calidad y cantidad. Las variables dependientes que se miden son la velocidad de recorrido y otras respuestas del sujeto que varían en función del cambio del reforzador, comparado con controles que no son expuestos a ellos. Además, se evalúan respuestas fisiológicas y neurales. En preparaciones consumatorias se mide el consumo, lameteos o tiempo de permanencia en el bebedero de soluciones apetitivas (e.g. agua azucarada en distintas concentraciones). En todas estas preparaciones predominan los procesos emocionales, a excepción del contraste anticipatorio y el conductual, en los cuales sobresalen los procesos 
cognitivos (Flaherty \& Rowan, 1985; Pérez, Díaz \& Pulido, 2009).

En los estudios experimentales con humanos se utilizan como incentivos juguetes, alimentos, restricción de movimientos o interrupción de tareas placenteras si son bebés o niños (Kobre \& Lipsitt, 1972; Miller \& Karniol, 1976); y exclusión social, realización de tareas difíciles o irresolubles, cambio de imágenes placenteras a neutras, perder en un juego, noticias de empeoramiento económico, entre otras, si son adultos (Cuenya, Kamenetzky, Fosacheca, \& Mustaca, 2013; Kamenetzky, Cuenya, Elgier, López Seal, Fosacheca, et al., 2009).

La mayoría de las teorías consideran que la frustración se asocia con respuestas conductuales, emocionales, psicofisiológicas y neurales similares o idénticas a la presentación de estímulos aversivos o su anticipación, por lo que se la considera un modelo de estrés y dolor psicológico (Amsel, 1958; Gray, 1987; Gray \& McNaughton, 2000; Konorsky, 1964, 1967; Mustaca, 2013; Papini, Fuchs \& Torres, 2015). Según Amsel (1992), la omisión o disminución sorpresiva de un reforzador positivo provoca activación o potenciación de cualquier comportamiento que le sigue inmediatamente al evento frustrante, llamado efecto de frustración $(\mathrm{EF})$. Se trata de una reacción incondicionada, aversiva, llamada frustración primaria, que ejerce un efecto motivacional parecido al drive o arousal. Es este estado lo que lleva a los animales a recorrer el laberinto más rápido (Amsel \& Roussel, 1952), ambular más, pararse en dos patas, escapar, etc., cuando se omite o disminuye un reforzador y respuestas neurales semejantes al dolor y estrés. Después de esta primera reacción, se produce un proceso de condicionamiento de las respuestas anticipatorias de la frustración primaria, llamada frustración secundaria o condicionada. Las respuestas que provoca son de conflicto y ansiedad, debido a que los estímulos están asociados con dos tipos de respuestas: apetitivas y aversivas. Finalmente, los animales suelen adaptarse y responder, como los sujetos, con controles no expuestos a situaciones de frustración. De esto se deduce que la frustración primaria y la secundaria pueden tener mecanismos distintos, como se evidenció en algunas investigaciones. Por otra parte, suelen ser reacciones transitorias. Sin embargo, esto no implica que situaciones de frustraciones muy intensas, como, por ejemplo, la pérdida de un trabajo o de un ser querido, no desencadenen en el organismo cambios duraderos. La teoría de Amsel sobre la frustración sigue siendo la más general y la que ha sido puesta a prueba en numerosos procedimientos experimentales. Más adelante, Gray (1987) se abocó a establecer las bases neurobiológicas del miedo y la frustración guiado por las teorías de Amsel y de Konorsky (1967) que afirman la existencia de una equivalencia funcional y neurobiológica entre la omisión de reforzadores apetitivos y la presentación de estímulos aversivos. Para validar esta hipótesis se utilizan métodos que se pueden sintetizar en evaluar si las respuestas conductuales, fisiológicas y neurales entre ambos eventos se solapan o son análogas. Estos métodos se pueden combinar entre sí y, aunque aún no hay una respuesta definitiva, los resultados apoyan la hipótesis propuesta (Mustaca et al., 2005; Mustaca, 2013; Papini, Fuchs \& Torres, 2015).

El objetivo de este artículo es presentar investigaciones que mostrarán cómo la violación de expectativas positivas influye sobre las respuestas sociales. Además, se mencionarán experimentos que utilizan estímulos aversivos con el objeto de comparar sus resultados y validar la teoría de equivalencia psicofisioneurobiólogica entre las dos clases de eventos. En la mayoría de las investigaciones sobre el tema, los sujetos son sometidos a estados de frustración en contextos que comparten con otros congéneres y se miden las respuestas sociales que desencadenan, comparándolas con grupos controles no frustrados. En estudios con humanos suelen administrarse diferentes pruebas psicométricas que dan cuenta de otros factores individuales que pueden influir en los resultados y no siempre miden las respuestas sociales ante 
congéneres, sino que utilizan otros métodos, como cuestionarios, vocabulario utilizado o toma de decisiones que indirectamente implican alteraciones en la conducta social, que se suele llamar agresividad implícita. En este artículo, por razones de espacio, se presentarán, principalmente, los cambios comportamentales provocados por la frustración, sin mencionar sus correlatos neurales o fisiológicos. Además, el artículo se restringirá, por las mismas razones, a los efectos de la frustración sobre la conducta agonística y a los de la privación social (frustración por pérdida de reforzadores sociales) sobre diferentes respuestas.

\section{Frustración y conducta agonística}

\section{Investigaciones con animales no humanos}

La conducta agonística se refiere a las respuestas sociales relacionada con la lucha. Incluye la agresión, el apaciguamiento, el escape, la evitación y patrones de amenaza inter o intraespecíficas.

La hipótesis según la cual la frustración causa agresión, al igual que la presentación de estímulos aversivos, tiene una larga tradición. Fue desarrollada inicialmente por Dollard, Dobb, Miller, Mower y Sears (1939) y más adelante por Berkowitz (1989), inspirados en el psicoanálisis. Para la teoría de Amsel (1992), la presencia de agresión después de la frustración primaria sería producto de la activación generalizada; emerge porque puede llegar a ser la primera en la jerarquía de respuestas posibles. La teoría sobre frustración-agresión tuvo tanta repercusión que Maier (1942), en pleno periodo de la segunda guerra mundial, asoció los estudios de laboratorio sobre el tema con la conducta social en la época del nazismo y con todas las sociedades autoritarias donde existe restricción de la libertad. Su teoría afirmaba la existencia de al menos dos clases de motivación: la dirigida hacia metas (relacionado con condicionamiento apetitivo) y la instigada por la frustración, que conlleva conductas de agresión, miedo y conflicto, entre otras (asociado con el condicionamiento aversivo y la pérdida de reforzadores apetitivos). A la vez, distinguió dos grandes movimientos sociales. El fascismo, que establece un capitalismo de estado totalitario, una economía dirigista y un nacionalismo con componentes revanchistas que conducen a la violencia y corporaciones de seguridad del régimen contra los que el estado define como enemigos, utilizando un eficaz aparato de propaganda y alto control. Sus líderes son personalistas y su función es mantener el orden con discursos agresivos, en el cual hay enemigos y amigos, lo que genera fanatismo, violencia y miedo. Afirmó que esta organización social se basa en mecanismos psicológicos relacionados con la motivación provocada por la frustración por la falta de libertad y discriminaciones, y por el miedo provocado por las amenazas y castigos por conductas opuestas al régimen. El segundo movimiento social que consideró es el de las democracias. Este sistema está formado por grupos organizados en torno a objetivos comunes y atractivos que variarán en distintos individuos. Sus líderes representan al grupo, están cerca de toda la sociedad y atentos a sus necesidades. En este movimiento predomina la motivación hacia metas y hacia la satisfacción de necesidades, existe mayor libertad y respeto por los individuos, más que a tener en cuenta ideas dogmáticas. Si bien esta extrapolación parece un tanto exagerada y es difícil ponerla a prueba de modo concluyente, son sugerentes y muestra que los investigadores de laboratorio, en la mayoría de los casos, no pierden la perspectiva de aplicar sus conocimientos para entender las situaciones cotidianas de las sociedades complejas. De todos modos, como se verá más adelante, algunas investigaciones apoyan la idea de que acontecimientos sociales relacionados con pérdidas o violación de expectativas pueden inducir, entre otros comportamientos, a la violencia.

Si bien, se mostró que no necesariamente la presentación de estímulos aversivos y la omisión de incentivos produce agresión, hay evidencia 
de que, bajo ciertas condiciones, la hipótesis se valida. Cuando se administran choques eléctricos en las patas de dos ratas enjauladas, inmediatamente empiezan a pelearse entre ellas (Dunlap, Gentry \& Zeigler, 1939). Estos resultados se replicaron en situaciones de frustración en una gran variedad de especies, como palomas, primates y peces (Azrin, Hutchinson \& Hake, 1966). Bajo programas de extinción apetitiva, los animales suelen desplegar respuestas agresivas si hay congéneres cerca (O’Kelly \& Steckle, 1939; Ulrich \& Azrin, 1962). Por ejemplo, Azrin et al. (1966) expusieron a unas palomas a periodos sucesivos de adquisición y extinción, mientras en la misma caja de condicionamiento había un congénere atado e inactivo. Cuando comenzaba el periodo de extinción, las palomas atacaban a su par. Los ataques ocurrían en la primera porción de la etapa de extinción, aumentaban monotónicamente en función del número de reforzamientos previos $\mathrm{y}$ se reducían cuando las palomas tenían libre acceso al alimento. Con ratas ocurrió lo mismo en un corredor lineal (Gallup, 1965), con palomas y ratas en la caja de Skinner (Davis \& Donenfeld, 1967) y, en chanchitos de Guinea, se observó además que la agresión estaba asociada con un aumento de corticosterona en su plasma sanguíneo, lo que indica un estado de estrés (Dantzer, Arnone \& Mormone, 1980). Davis y Donenfeld (1967) mostraron que, cuando a las ratas se les sacaba el refuerzo en una caja de Skinner, aumentaban las agresiones a otras en función de la extinción de cada par, exhibían desde posiciones de amenazas hasta la expulsión de la caja.

La respuesta agresiva se extiende a señales que predicen ausencia de refuerzo. Por ejemplo, Tomie, Carelli y Wagner (1993) mostraron, en ratas sedientas, que una correlación negativa entre un tono y agua aumentaba las mordidas a un objeto durante la presencia del tono si previamente el tono se había presentado inmediatamente antes del agua. Del mismo modo, en salmones del Atlántico, se halló que ante la presencia de estímulos condicionados que predicen ausencia de reforzadores, los animales despliegan respuestas agresivas a sus congéneres (Vindas et al., 2012). Por otra parte, cuando los monos Rhesus fueron aislados de su grupo social y se los entrenó posteriormente en un programa de reforzamiento y extinción, manifestaron conductas transitorias de autoagresión en las etapas de extinción. Esta conducta fue transitoria, apareció en las primeras transiciones de condicionamiento apetitivo-extinción, y luego desapareció (Gluck \& Sackett, 1974). Es posible que la disminución de la autoagresión se podría haber reducido por contracondicionamiento de la activación generalizada (el concepto de contracondicionamiento se describirá en el apartado resumen y conclusiones).

Aunque en la mayoría de los casos se halló que el miedo y la frustración genera agresión, también se obtuvieron resultados opuestos. En las preparaciones de indefensión aprendida (Overmier \& Seligman,1967) es donde más se ha mostrado que los animales expuestos a choques eléctricos incontrolables e impredecibles mostraban conductas de sometimiento más que de agresión. Williams (1987) y Williams y Lierle (1986) hallaron que las ratas dominantes expresaban patrones de sumisión después de recibir choques eléctricos incontrolables. Además, el efecto desaparecía si previamente a la presentación de choques inevitables, los animales eran expuestos a un entrenamiento de condicionamiento apetitivo instrumental. Los resultados de disminución de la agresividad se replicaron en dos preparaciones de frustración, el contraste sucesivo negativo consumatorio (CSNC) y la extinción consumatoria (EC). En el CSNC los animales expuestos a la devaluación de una solución azucarada (e.g., $32 \%$ a $4 \%$ ) tienen una disminución abrupta del consumo respecto de controles que siempre consumieron la solución al $4 \%$, que se torna igual a los del grupo control después de unos 3-4 ensayos (Flaherty, 1996). Esta respuesta viene acompañada con mayor ambulación y elevamientos de las patas delanteras (ver en Youtube contraste sucesivo negativo 
consumatorio), y otras repuestas conductuales y neurofisiológicas similares a las de dolor y miedo (Mustaca, 2013; Papini \& Dudley, 1997; Papini, Fuchs \& Torres, 2015). En la EC, el reforzador se omite completamente (Mustaca, Freidín \& Papini, 2002). Respecto de la respuesta social, se halló en varios experimentos que, cuando inmediatamente después de la devaluación del reforzador o de la EC, los animales se exponen a otro congénere, expresan patrones comportamentales de sumisión y de pérdida de la dominancia (Mustaca \& Martínez, 2000; Mustaca, Martínez, \& Papini, 2000), un resultado similar a los obtenidos cuando reciben choques eléctricos incontrolables y opuesto a los encontrados anteriormente cuando se utilizaron procedimientos instrumentales.

\section{Investigaciones con humanos}

En humanos, al igual que en los otros animales, si bien los resultados tendieron a mostrar que la frustración genera agresión, las medidas dependientes utilizadas pueden dar lugar a interpretaciones ambiguas, en otros casos no desencadena respuestas agresivas. Existen razones éticas para evitar diseños que implique daño físico en los sujetos. Los investigadores, conscientes de esta dificultad, suelen utilizar medidas de agresiones implícitas, como uso de malas palabras, escalas de agresividad y castigos verbales o punitorios. Además, los experimentos no pueden controlar la historia de aprendizaje ni las diferencias individuales por razones genéticas, que pueden modular las respuestas. Como contrapartida a estas limitaciones para evaluar relaciones causales entre frustración y conducta social, se pueden medir factores de personalidad u otros rasgos que modulen estas relaciones (Frederiksen \& Peterson, 1977).

Nation y Cooney (1982) proporcionaron a un conjunto de estudiantes dos formas de escapar de ruidos intensos y azarosos mientras hacían una tarea manual que se recompensaba con fichas: presionando suavemente un botón o golpeando una plataforma que requería hacer una fuerza mínima de 25 libras $(11,34 \mathrm{~kg}$ ). Se halló que cuando recibían recompensa por la tarea, los sujetos, para escapar del ruido, pulsaban el botón; en cambio, cuando no se los reforzaban (extinción), golpeaban la plataforma que requería mayor energía. Esta elección después de la omisión de la recompensa se interpretó como una expresión de agresión; sin embargo, no se puede descartar que refleje simplemente una conducta más enérgica, típica de la reacción de activación provocada por la extinción.

Los impactos de la observación de videojuegos sobre la agresividad, que comenzaron con Bandura (1974), son fuente de controversia, ya que no hay resultados concluyentes sobre el tema (De la Torre-Luque \& Valero-Aguayo, 2013). Algunos estudios recientes se concentran también en evaluar la hipótesis frustración-agresión mediante diseños experimentales con videojuegos. Por ejemplo, Breuer, Scharkow y Thorsten (2015), con estudiantes, manipularon las condiciones experimentales de modo que haya perdedores y ganadores en un juego de futbol en línea. A continuación de la terminación del juego, se les decía que iban a competir en otro tipo de tareas con los equipos ganadores, en el cual, cuando los sujetos fallaban, los podían castigar con un ruido de distintos volúmenes. Hallaron que los sujetos que perdieron en el juego anterior, cuando tenían oportunidad de castigar, elegían hacerlo con el mayor volumen posible, a diferencia de los que habían ganado, que presionaban a un volumen más bajo. Además, esa respuesta se halló mediatizada por los puntajes obtenidos en una escala de afectividad negativa que llenaban después de terminar con el juego de fútbol: cuanto mayor puntuaban en afectividad negativa, mayor era el volumen que utilizaban para el castigo. Por otra parte, esa respuesta no estuvo influida por los comentarios neutros o irónicos del experimentador cuando los sujetos perdían en el primer juego, ni tampoco por el género de los participantes. Este diseño es muy interesante, aunque faltaría la comparación con un grupo experimental "empate", ya 
que, al comparar la mayor agresividad implícita en los perdedores, no se puede descartar que los resultados no hayan sido producto de la afectividad positiva de los ganadores, más que por la frustración de los perdedores.

Relacionado con la investigación previa, son frecuentes las noticias sobre la violencia que desencadena el fútbol y otros deportes, casi siempre realizada por grupos de fanáticos de los equipos que perdieron. Gantz, Bradley y Wang (2006), Rees y Schnepel (2009), Priks (2010) y Card y Dahl (2011) encontraron mayores índices de violencia relacionado con el deporte. En el mismo sentido, Munyo y Rossi (2013) idearon un estudio de campo en Uruguay con alta validez ecológica que indirectamente apoya la hipótesis de la asociación entre frustración y agresión. Compararon las denuncias de delitos antes, durante y después de partidos de fútbol de los dos equipos favoritos en Uruguay: Peñarol y Nacional, durante 8 años. Para conocer cuál era el equipo favorito en cada partido (expectativa de éxito), se definía como el que recibía más apuestas en cada partido. Hallaron que en los partidos que perdía el favorito (violación de la expectativa) aumentaban los delitos durante una hora después de terminado el partido, comparado con los denunciados una hora antes del partido. Estos hechos violentos se registraron en toda la región, no solo en las cercanías del lugar donde se realizaba el encuentro. La contrapartida fue que, si ganaba el equipo favorito, los delitos disminuían.

Los diseños experimentales con humanos permiten evaluar si ciertas características personales interactúan con la relación entre la frustración y la violencia. En ese sentido, Wang y Zhong (2015) presentaron un experimento con estudiantes de pregrado donde eran o no sometidos a una situación de frustración a sujetos a los cuales previamente los evaluaron con una prueba de resiliencia. La medida de agresividad implícita la medían por el vocabulario que usaban cuando eran frustrados (número de palabras neutras o agresivas). Hallaron que el vocabulario agresivo se manifestó solamente en los sujetos frustrados con baja resiliencia. Los autores relacionaron este efecto como un indicador de que las personas con baja capacidad de recuperación cuando tienen sucesos frustrantes son más proclives a tener vocabularios agresivos que aquellos con alta capacidad de recuperación.

Otros estudios hallaron correlaciones positivas entre cambios económicos negativos (que se pueden considerar pérdidas o disminuciones de incentivos) y mayor cantidad de actos violentos y delincuencia. Catalano, Novaco y McConnell (2002), Catalano, Snowden, Shumway y Kesse11 (2007) y Yoon y Joo (2005) mostraron que el aumento de la tasa de desempleo estaba positivamente relacionado con indicadores de violencia y Landau (1997) mostró una relación negativa entre la percepción subjetiva de la situación económica de la población (los puntajes altos indicaban percepción más positiva) y las tasas de homicidios de hombres y mujeres. Barlett y Anderson (2014) hicieron completar, por estudiantes universitarios escalas y medidas de agresividad, rasgos de hostilidad y percepción de estrés por crisis económicas. Encontraron correlaciones positivas entre la mayor percepción del estrés por cambios negativos en la sociedad y los puntajes en las escalas de agresión, mediatizadas por rasgos de hostilidad. Para evaluar si la percepción de crisis social era la causa del aumento de los puntajes de agresividad y hostilidad, idearon un diseño experimental en el cual dividieron al azar a los estudiantes para que observaran, alrededor de $5 \mathrm{~min}$, dos clases de situaciones: videos con noticias que indicaban la presencia de una economía pobre y en crisis (grupo 1), o videos con informaciones neutras (grupo 2). Inmediatamente después llenaban cuestionarios de rasgos de agresividad, percepción de estrés, arousal y hostilidad. Los resultados mostraron que el estrés correlacionó negativamente con percepción de arousal y positivamente con la hostilidad; a la vez, esta última correlacionó positivamente con rasgos de agresividad. También se hallaron 
diferencias entre varones y mujeres: los primeros tuvieron puntajes más altos de agresividad y percepción de arousal que las segundas. En cuanto a la comparación entre los dos grupos, se halló que aquellos estudiantes que vieron el video sobre crisis económica, puntuaron significativamente más en hostilidad, agresividad y niveles de estrés que los expuestos al video neutro. Este experimento muestra que breves observaciones de situaciones de pobreza económica causan un aumento de estrés y rasgos de agresividad que pueden llegar a provocar estados de hostilidad y conductas agresivas, aunque ello no necesariamente la determina. Con base en estos y otros estudios, Anderson y Bushman (2002) postularon un modelo de agresividad general (GAM). El modelo afirma que la personalidad y las variables situacionales interactúan para generar estados afectivos, cognitivos y de excitación fisiológica que pueden conducir a que se desencadenen procesos de evaluación y toma de decisiones que pueden llevar a comportamientos reflexivos o impulsivos, por lo que los sujetos pueden ser agresivos o no. Este modelo de agresividad estaría relacionado con lo propuesto en la teoría de la frustración de Amsel (1992) y de Berkowitz (1989); la frustración sería uno de los tantos eventos que pueden provocar activación generalizada.

Los estudios descritos anteriormente apoyan indirectamente la teoría de Maier (1944). Sin embargo, hay que tener en cuenta que estos diseños son algunos correlacionales, otros miden respuestas relacionadas con la violencia, y la mayoría las evalúan inmediatamente después de la frustración. Además, la variable de interés de esta investigación (frustración) se infiere de las observaciones que realizan los sujetos sobre situaciones negativas, que podrían desencadenar respuestas de estrés pero no de violación de expectativas.

Relacionado con lo anterior, otros resultados no siempre hallaron asociaciones entre eventos de crisis o pobreza con la violencia, sino de adaptación o resignación. Tironi (1989), en un largo estudio descriptivo de psicología política, menciona que la deducción, según los paradigmas "la pobreza induce a la frustración" "la frustración conduce a la violencia", de que la pobreza induce a la violencia es un mito:

[...] se deriva que, en la medida en que ven parcialmente satisfechas sus necesidades socio-económicas, los grupos más acomodados (en particular las clases medias) tenderían a rechazar el uso de la violencia; la predisposición hacia la violencia sería por tanto un atributo peculiar de los grupos más pobres (p. 9).

Además, presenta una investigación realizada en Chile, en la cual utiliza un cuestionario con escalas tipo Likert, que mide actitudes de la orientación hacia la violencia, índice de frustración, adaptación/resignación, individualismo, politización, autoagresividad y coerción en sujetos que viven en zonas marginales, a los que llama pobladores y de clase media. Sintéticamente, halló que los pobladores no se caracterizaron por índices elevados de orientación a la violencia ni de frustración; entre estos y la clase media no hubo un patrón netamente diferenciado respecto a la violencia; y mientras que la frustración se incrementó levemente con la pobreza, la predisposición a la violencia tendió más bien a disminuir. Además, se observó un índice de adaptación /resignación más alto en el grupo de pobladores que en los de clase media y menor frustración, menor tendencia a la violencia, mayor individualización y menor politización. Aunque este estudio es metodológicamente limitado, ya que es descriptivo, el cuestionario no está validado y en sus tablas muestra porcentajes de respuestas sin utilizar estadísticas inferenciales, se presenta en esta revisión. Se presenta en esta investigación debido a que tiene un valor heurístico, sugiere que hay que diferenciar y delimitar mejor el concepto de frustración cuando se analizan situaciones sociales más complejas. De hecho, la pobreza o las situaciones crónicas aversivas pueden relacionarse más con los protocolos de estrés crónicos o de 
indefensión aprendida que con los de frustración. Se debe recordar que el fenómeno de la indefensión aprendida se manifiesta por medio de tres déficits: (i) motivacional: retraso en la iniciación de respuestas voluntarias; (ii) cognitivo: dificultad en aprender posteriormente que una respuesta controla una consecuencia, cuando previamente no la ha controlado, y (iii) emocional, desórdenes conductuales y fisiológicos característicos de un estado de ansiedad y miedo seguido de depresión. Otra posibilidad, quizá más plausible, es que situaciones crónicas de pobreza conduzcan a un proceso de habituación que hace que los individuos se adapten a esas situaciones adversas.

\section{Efectos del aislamiento social y exclusión social}

\section{Estudios con animales no humanos}

Se hizo referencia en la introducción cómo las pérdidas de reforzadores sociales provocan ansiedad, estrés y mayor vulnerabilidad a contraer enfermedades. El aislamiento social temprano, como así también aplicaciones de estímulos aversivos, en animales, y sus efectos sobre la adultez es otro de los protocolos que ha sido estudiado intensamente. En un principio, se mostró que el estrés en la infancia producía alteraciones conductuales y neurofisiológicas de mayor ansiedad y estrés ante situaciones específicas. Los clásicos experimentos de Harlow de la década de los cincuenta mostraron las dramáticas consecuencias que exhibieron los monos adultos criados en aislamiento (Harlow, 1958). Sin embargo, los resultados hallados más recientemente mostraron que los efectos son variados. Los sujetos que recibieron estímulos aversivos y de aislamiento social en la infancia, en la adultez pueden ser más ansiosos e inestables o más resilientes que los sujetos controles criados en condiciones normales. El resultado depende de una compleja interacción entre factores que incluyen tipos de estimulación, genéticos, epigenéticos y de aprendizaje (Cuenya, Kamenetzky \& Mustaca, 2014). Ruetti, Justel, Mustaca, Torrecilla y González Jatúff (2010) evaluaron el efecto de CSNC y de EC en ratas adultas sometidas a un protocolo de estrés crónico neonatal, donde se incluía aislamiento social. Hallaron que los animales expuestos a estrés neonatal presentaron una respuesta atenuada de frustración y, en la EC, una extinción más acelerada, en relación con sus controles sin tratamiento neonatal. Estos resultados fueron similares a los hallados con el mismo protocolo cuando se aplicaron pruebas que implicaban presentación de estímulos aversivos.

Entre los cambios producidos por el aislamiento en la adolescencia en ratas, aplicado desde el primer día de destete (a los 21 días de su nacimiento) hasta el día 30, se encuentra en la adultez una reducción de interacciones sociales, deterioro de mecanismos inhibitorios, expresados en menor inhibición latente e inhibición prepulso, hiperreactividad ante ambientes novedosos, inflexibilidad cognitiva, hiperactividad dopaminérgica en núcleo acumbens (NAC) y estriado ventral e hipoactividad glutamatérgica y dopaminérgica en la corteza prefrontal (Fone \& Porkess, 2008). Respecto de la frustración, Cuenya, Fosacheca, Mustaca y Kamenetzky (2011) y Cuenya, Fosacheca, Mustaca \& Kamenetzky (2012) evaluaron ratas aisladas en la adolescencia y hallaron que en la adultez no hubo diferencias en el efecto de CSNC. Sin embargo, exhibieron un aumento del efecto de contraste sucesivo positivo consumatorio (CSPc, Flaherty, 1996), comparado con los controles que permanecieron agrupados. En este procedimiento los animales que reciben dos ensayos sucesivos de $32 \%$ o $4 \%$ de soluciones azucarada consumen transitoriamente (en dos ensayos) más soluciones al $32 \%$ que aquellos que siempre consumen $32 \%$. Se lo considera un efecto opuesto a la violación de expectativas positivas, o sea un modelo de euforia (Flaherty, 1996). Los animales aislados en la adolescencia exhiben un efecto de contraste positivo durante 7 ensayos, 
mientras que sus controles, solamente en dos ensayos (Cuenya, Kamenetzky, \& Mustaca, 2015).

\section{Estudios con humanos}

Más recientemente, se realizaron experimentos con humanos que estudiaron las respuestas comportamentales, psicofisiológicas y neurales cuando los sujetos se exponían a condiciones de exclusión social o de rechazo social (Kamenetzky et al., 2009, Zadro \& Gonsalkorale, 2014). Las personas socialmente excluidas presentan reacciones de depresión, ansiedad, sentimientos de soledad y desamparo (Williams, Cheung \& Choi, 2000; Eisemberger, Jarcho, Lieberman \& Nalebuff, 2006). Respecto a los efectos sobre respuestas interpersonales, la exclusión social puede provocar disminución de respuestas prosociales, altruistas, aumenta la probabilidad de que los sujetos apliquen multas o castigos a personas que comenten delitos mucho más intensos que los sujetos no excluidos y la hostilidad hacia otras personas. Por ejemplo, Twenge, Baumeister, De Wall, Ciarocco y Bartels (2007) realizaron 7 experimentos en los cuales manipulaban la exclusión social diciéndoles a los estudiantes que, por unos test que le tomaban previamente, predecían que iban a pasar toda la vida solos y aislados o que otros participantes lo rechazaban (grupos exclusión). A otros estudiantes les decían lo contrario (grupo control). Los estudiantes que recibieron predicciones de exclusión donaron menos dinero a un fondo para estudiantes con pocos recursos, no estaban dispuestos a ser voluntarios para otros experimentos de laboratorio, fueron menos útiles después de un accidente y cooperaron menos en un juego posterior donde participaban otros estudiantes. Aunque en este diseño faltaría un grupo control en el cual a los sujetos no se les diera ninguna predicción acerca de su futuro a nivel social, muestra el impacto que tiene una noticia negativa sobre una futura pérdida de reforzadores sociales sobre respuestas prosociales.
Además de las respuestas mencionadas anteriormente, se ha mostrado que los sujetos excluidos pueden aumentar conductas que tienen a conectarse con los demás, como forma de volver a sentirse incluidos. Maner, DeWall, Baumeister y Schaller (2007) mostraron que las personas socialmente excluidas realizan conductas que les da oportunidad de relacionarse con otras personas, por ejemplo, hacer nuevas amistades, trabajar con otras personas, etc. Además, Mead, Baumeister, Stillman, Rawn et al. (2011) mostraron que sujetos que habían sido excluidos en una tarea pueden afectar sus respuestas como consumidores. Ellos son más propensos a comprar un producto simbólico que pertenece a un grupo específico, aunque no sean prácticos ni para un regalo, a adaptar sus preferencias de compra a las de otro asociado con él, a gastar dinero en un poco atractivo influenciado por un compañero y a informar que estaría dispuesto a probar una droga ilegal, si solo al hacerlo aumentara sus posibilidades de iniciar las conexiones sociales. Estos resultados sugieren que las personas socialmente excluidas pueden llegar a sacrificar su bienestar personal y financiero por el deseo de sentirse incluido.

\section{Resumen y conclusiones}

No es sorprendente que los estados emocionales influyan sobre las conductas. En este artículo se presentaron algunas evidencias relacionadas con la interacción entre la frustración y algunas conductas sociales. Desde el punto de vista teórico, los datos corroboran la hipótesis general que afirma que la presentación de estímulos aversivos y su anticipación y la omisión o pérdida de reforzadores apetitivos y su anticipación activan respuestas conductuales que implican mecanismos psicofisiológicos y neurales análogos. Estos procesos están relacionados con el dolor, el miedo y la ansiedad.

Respecto de la conducta agonística, tanto la presentación de estímulos aversivos como la frustración provoca activación generalizada, que 
conduce, en la mayoría de los casos, a respuestas violentas, de agresividad $\mathrm{u}$ otras relacionadas con ellas. Sin embargo, la regla que la frustración siempre provoca agresividad, desarrollada por Dollard et al., (1939), no es correcta (Berkowitz, 1987). Incluso en los diseños con modelos animales, relativamente más sencillos, se han encontrado resultados opuestos. Por ejemplo, en casi todos esos estudios se halló que los animales respondían con mayor agresividad cuando eran expuestos a la omisión o decremento de reforzadores, utilizando procedimientos instrumentales. En cambio, en los procedimientos consumatorios, como en el CSNC y la EC, las ratas exhibieron respuestas de sumisión ante congéneres. Estas disociaciones entre los procedimientos instrumentales y consumatorios se observó en otros fenómenos paradójicos del reforzamiento (Mustaca, Bentosela, Ruetti, Kamenetzky, Cuenya et al., 2009). Aún se desconoce cuáles son las condiciones necesarias y suficientes para la inducción de violencia o de sumisión ante la violación de expectativas; se deberá explorar en futuras investigaciones. Sin embargo, hay evidencias que, tanto la frustración como los eventos aversivos, pueden crear inclinaciones hacia la violencia, mediatizada por la activación generalizada o una afectividad negativa, pero que puede inhibirse por cuestiones culturales, de personalidad, situacionales, del contexto o de aprendizajes previos. Por lo cual, la hipótesis frustración $\longrightarrow$ agresividad, si bien fue confirmada en muchas investigaciones, como se presentó en el apartado de este artículo, tienen una vigencia relativa, no solo con diseños experimentales, sino también cuando se extrapola a contextos sociales o políticos.

La mayoría de los experimentos sobre frustración y sus relaciones con respuestas sociales mostraron las consecuencias inmediatas de los sujetos ante la violación de expectativas; no se encontraron datos que muestren si ellas perduran en el tiempo. Dado que las investigaciones de laboratorio indican que los efectos de la frustración, en especial en la frustración primaria, son transitorios, se podría suponer que también las consecuencias sobre la interacción social sean momentáneas, aunque deberían ponerse a prueba.

Otra cuestión que se exploró muy poco es cómo influye la historia de aprendizajes previos, las diferencias individuales y variables genéticas sobre las relaciones entre la interacción social y la frustración. Ya se mostró que los paradigmas de los efectos del aislamiento social o de estímulos aversivos en la infancia provocan en la adultez resultados que dependen de la interacción entre factores ambientales y biológicos. En cuanto a las diferencias individuales, se mostró que, en parte, las respuestas a la frustración están reguladas por los genes (Flaherty, 1994; Cuenya, Sabariego, Donaire, Fernandez-Teruel, Torres, \& Papini, 2015). Aunque no se evaluaron si las reacciones diferenciales en ratas endocriadas se extienden a los efectos de la frustración sobre las respuestas sociales, es posible conjeturar que ellas deberían influir: otro vacío en la información que debiera llenarse.

Respecto de los factores ambientales, se estudió extensamente, en modelos animales, cómo los programas de reforzamiento intermitente, donde una respuesta se adquiere recibiendo de modo azaroso $50 \%$ reforzamiento positivo y $50 \%$ choques eléctricos suaves, retraso en el refuerzo o no refuerzo, produce a posteriori una mayor resistencia a la extinción (Amsel, 1992; Brown \& Wagner, 1964), en comparación con el reforzamiento continuo. Según la teoría de Amsel (1992), los sujetos sometidos a reforzamiento intermitente aprenden por contracondicionamiento a que, tanto la presencia (respuesta de acercamiento) como la ausencia o disminución del reforzador apetitivo (respuesta de alejamiento y estado interno de estrés) están señalados por los mismos estímulos condicionados. El conflicto se resuelve a favor de responder, de modo que la conducta persiste cuando se introduce la extinción, ya que la respuesta se ha condicionado a la expectativa de ausencia o disminución de la de recompensa. En cambio, en los sujetos reforzados 
continuamente no hay nada que los motive a responder en ausencia de recompensa. Es así que los programas de reforzamiento intermitente generan persistencia, valentía y mayor tolerancia al dolor psicológico o sensorial que perduran, pueden repetirse en distintos contextos, situaciones y respuestas que Amsel (1992) denominó aprendizaje y memoria disposicional. Desde el punto de vista teórico se puede predecir que los procesos asociativos de contracondicionamiento deberían producir un decremento en la activación generalizada, que provocaría una reducción de todas las reacciones a la frustración. Esta hipótesis fue confirmada en numerosos procedimientos de frustración (Pellegrini, Muzio, Mustaca \& Papini, 2004) y es usado en la práctica clínica y educacional para generar. Sin embargo, de lo relevado en la bibliografía, no se evaluó si el efecto de reforzamiento intermitente podría modificar las respuestas ante situaciones sociales. Sería de interés, tanto básico como aplicado, averiguar si el reforzamiento intermitente puede modular las consecuencias de la frustración sobre la interacción social. Algunos trabajos que se mencionaron en esta recopilación apoyan estas ideas de modo indirecto (Wang \& Zhong, 2015).

En resumen, las investigaciones sobre las interrelaciones entre la conducta social y la frustración siguen apoyando la equivalencia funcional entre la presentación de estímulos aversivos y la omisión de reforzadores. Ambas condiciones provocan cambios en respuestas sociales, aunque ellas son diversas, dependiendo de múltiples factores, lo que impide realizar predicciones precisas. Los experimentos futuros deberían ahondar más en el estudio de la duración de las alteraciones halladas, así como la influencia de la historia de aprendizajes previos, de las diferencias individuales y sus bases neurofisiológicas. La posibilidad que se tiene actualmente de acceder no solo a estudiar las consecuencias comportamentales de la frustración, sino también las estructuras cerebrales y neurales, y hasta de medir la actividad de neuronas específicas asociadas con ellas, está abriendo un amplio campo para realizar estudios interdisciplinarios que, a largo plazo, desarrollen una teoría integrada de los componentes conductuales y neurofisiológicos implicados en la violación de expectativas.

\section{Referencias}

Amsel, A. (1958). The role of frustrative nonreward in noncontinuous reward situation. Psychological Bulletin, 55, 102-119.

Amsel, A. (1992). Frustration theory. Cambridge, UK: Cambridge University Press. Appleton. Traducción al castellano en Madrid: Alianza, 1984.

Armetz, B. B., Wasserman, J., Petrini, B., Brenner, S. O., Levy, L., Eneroth, P. et al. (1987). Immune function unemployed women. Psychosomatic Medicine, 49, 3-12.

Azrin, N. H., Hutchinson, R. R., \& Hake, D. F. (1966). Extinction induced aggression. Journal of the Experimental Analysis of Behavior , 9, 191-204.

Barlett, C. P., \& Anderson, C. A. (2014). Bad News, Bad Times, and Violence: The Link Between Economic Distress and Aggression. Psychology of Violence, 4, 309-321.

Bartels, J. M. (2007). Social Exclusion Decreases Prosocial Behavior. Journal of Personality and Social Psychology, 92, 56-66.

Batrop, R. Lazarus, L., Luckhurst, E., Kiloh, L. G., \& Penny, R. (1977). Depressed lymphocyte function after bereavement. Lancet, 1, 834-836.

Berkowitz, L. (1989). Frustration-aggression hypothesis: Examination and reformulation. Psychological Bulletin, 106, 59-73.

Breuer, J., Scharkow, M., \& Thorsten, Q. (2015). Sore Losers? A Reexamination of the Frustration-Aggression Hypothesis for Colocated Video Game Play. Psychology of Popular Media Culture, 4, 126-137.

Brown, R. T., \& Wagner, A. R. (1964).Resistance to punishment and extinction following training with shock or nonreinforcement. Journal of Experimental Psychology, 68, 503-507. 
Card, D., \& Dalg, G. (2011). Family violence and football: the effect of unexpected emotional cues on violent behavior. Quarterly Journal of Economics, 126, 103-143.

Catalano, R., Novaco, R. W., \& McConnell, W. (2002). Layoffs and violence revisited. $A g$ gressive Behavior, 28, 233-247.

Catalano, R., Snowden, L., Shumway, M., \& Kessell, E. (2007). Unemployment and civil commitment: A test of the intolerance hypothesis. Aggressive Behavior, 33, 272-280.

Cuenya, L., Fosacheca, S., Mustaca, A., \& Kamenetzky, G. (2011). Efectos del aislamiento en la adultez sobre el dolor y la frustración. Psicológica, 32, 49-63.

Cuenya, L., Fosacheca, S., Mustaca, A., \& Kamenetzky, G. (2012). Effects of isolation in adulthood on frustration and anxiety. Behavioural Processes, 90, 155-60.

Cuenya, L., Kamenetzky, G., \& Mustaca, A.E. (2014). Early experience and incentive relativity in adulthood. International Journal of Comparative psychology, 27, 459-473.

Cuenya, L., Kamenetzky, G., Fosacheca, S., \& Mustaca, A. (2013). Efecto del contraste sucesivo negativo en la valoración emocional de imágenes. Anales de psicología, 2, 944-952.

Cuenya, L., Mustaca, A., \& Kamenetzky, G. (2015). Postweaning isolation affects responses to incentive contrast in adulthood. Developmental Psychobiology, 27, 1-12.

Cuenya, L., Sabariego, M., Donaire, R., Fernández-Teruel, A., Torres, C., \& Papini, M. R. (2015). Transfer across reward devaluation tasks in inbred Roman rat strains. Learning \& Motivation, 52, 22-31.

D’Aquila, P. S., Brain, P., \& Willner, P. (1994). Effects of chronic mild stress on performance in behavioural tests relevant to anxiety and depression. Physiology \& Behavior, 56, 861-867.

Dantzer, R. Arnone, M., \& Mormone, P. (1980). Effects of frustration on behavior and plasma corticoesteroid levels in pigs. Physiology and Behavior, 24, 1-4.

Davis, H. \& Donenfeld. I. (1967). Extintion induced social interaction in rats. Psychonomic Science, 7, 85-86.

De la Torre-Luque, A., \& Valero-Aguayo (2013). Factores moduladores de la respuesta agresiva tras la exposición a videojuegos violentos. Anales de psicología, 29, 311-318.

Dollard, J., Dobb, L. W., Miller, N. E., Mower, O. H., \& Sears, R. R. (1939). Frustration and agression. New Haven, CN: Yale University Press.

Doob, A. N., \& Kirshenbaum, H. M. (1973). The effects of arousal of frustration and aggressive films. Journal of Experimental Social Psychology, 9, 57-654.

Dunlap, K., Gentry, E., \& Zeigler, T. (1931). The behavior of white rats under food and electric shock stimulation. Journal of Comparative Psychology, 12, 371-378.

Eisemberger, N., Jarcho, J., Lieberman, M., \& Nalebuff, B. (2006). An experimental study of shared sensitivity to physical pain and social rejection. Pain, 126, 132-138.

Flaherty, C. F. (1996). Incentive relativity. Cambridge, UK: Cambridge University Press.

Flaherty, C. F., Becker, H. C., \& Pohorecky, L. (1985). Correlation of corticosterone elevation and negative contrast varies as a function of postshift day. Animal Learning and Behavior, 13, 309-314.

Flaherty, C. F., Krauss, K. L., Rowan, G. A., \& Grigson, P. S. (1994). Selective Breeding for Negative Contrast in Consummatory Behavior. Journal of Experimental Psychology. Animal Behavior Processes, 20(1), 3-19.

Fone, K. C. F., \& Porkess, M. P. (2008). Behavioural and neurochemical effects of post-weaning social isolation in Rodents-Relevance to developmental neuropsychiatric disorders. $\mathrm{Neu}$ roscience and Biobehavioral Reviews, 32(6), 1087-1102. 
Frederiksen, L. W., \& Peterson, G. L. (1977). Schedule-induced aggression in humans and animal: A comparative parametric review. Aggressive Behavior, 3, 57-75.

Gallup, G. G. (1965). Aggression in rats as a function of frustrative nonreward in a straight alley. Psychonomic Science, 3, 99-100.

Gantz, W., Wang, Z., \& Bradley, S.D. (2006). Televised NFL games, the family, and domestic violence, En A. Raney and J. Bryant (Eds.). Handbook of Sports and Media, (pp. 365-382). Mahwah, NJ: Erlbaum.

Gluck, J. P., \& Sackett, G. P. (1974). Frustration and self-aggression in social isolate rhesus monkeys. Journal of Abnormal Psychology, 83, 331-334.

Gray, J. A., \& McNaughton, N. (2000). The Neuropsychology of Anxiety: An Enquiry into the Functions of the Septo-Hippocampal System (2 ed.) Oxford Psychology Series No. 33. Oxford: Oxford University Press.

Gray, J. A. (1987). The Psychology of fear and stress. Cambridge, UK: Cambridge University Press.

Gutiérrez-Cuesta, J. Martín-García, E., \& Maldonado, R. (2012): Operant model of frustrated expected reward in mice. Addiction Biology, 17, 770-782.

Hall, M., \& Irwin, M. (2001). Physiological indices of functioning in bereavement. In M. S. Stroebe \& R. O. Hansson (Eds.), Handbook of bereavement research: Consequences, coping, and care (pp. 473-492). Washington, DC: American Psychological Association.

Harlow, H. F. (1958). The Nature of Love. American Psychologist, 13, 673-685.

Holmes, T. H., \& Rahe, R. H. (1967). The social re-adjustment rating scale. Journal of Psychosomatic Research, 11, 213-218.

Kamenetzky, G. V., Cuenya, L., Elgier, A. M, López Seal, F., Fosacheca, S., Martin, L., \& Mustaca, A. E. (2009). Respuestas de frustración en humanos. Terapia Psicológica, 27, 2, 191-201.

Kavaliers, M. (1988). Brief exposure to a natural predator, the shot-tailed weasel, induces ben- zodiazepine-sensitive analgesia in white-footed mice. Physiology and Behavior, 43, 187-193.

Kielcolt-Glaser, J. K., Kennedy, S., Malcoff, S., Fisher, L., Speicher, C. E., \& Glaser, R. (1988). Marital discord and immunity in males. Psychosomatic Medicine, 50, 213-229.

Kobre, K. R., \& Lipsitt, L.P. (1972). A negative contrast effect in newborns. Journal of Experimental Child Psychology, 14, 81-91

Konorsky, J. (1967). Integrative activity of the brain. Chicago: University of Chicago Press.

Landau, S. F. (1997). Crime patterns and their relation to subjective social stress and support indicators: The role of gender. Journal of Quantitative Criminology, 13, 29-56.

Maier, N. R. F. (1942). The role of frustration in social movements. Psychological Review, (49)6, 586-599.

Maner, J. K., DeWall, C. N., Baumeister, R. F., \& Schaller, M. (2007). Does social exclusion motivate interpersonal reconnection? Resolving the "porcupine problem". Journal of Personality and Social Psychology, 92, 42-55.

Mead, N. L., Baumeister, R. F., Stillman, T. F., Rawn, C., \& Vohs, K. (2011). Social Exclusion Causes People to Spend and Consume Strategically in the Service of Affiliation. Journal of Consumer Research, 37, 902-919.

Miller, D., \& Karniol, R. (1976). Coping strategies and attentional mechanisms in self-imposed and externally imponed delay situations. Journal of Personality and Social Psychology, 34, 310-316.

Munyo, I., \& Rossi, M. (2013). Frustration, Euphoria, and Violent Crime. Journal of Economic Behavior and Organization, 89, 136-142.

Mustaca, A. (2001). Emociones e Inmunidad. Revista Colombiana de Psicología, 10, 5-14.

Mustaca, A. (2013). "Siento un dolor en el alma": ¿metáfora o realidad? Revista Argentina de Ciencias del comportamiento, 5, 2, 47-60.

Mustaca, A., Bentosela, M., Pellegrini, S., Ruetti, E, Kamenensky, G., \& Cuello, M. (2005). Aportes 
para la comprensión de la frustración. En J. Vivas (Comp.), Las Ciencias del Comportamiento en los albores del Siglo XXI (pp. 313-322). Argentina: Editorial Universidad Nacional de Mar del Plata.

Mustaca, A., \& Bentosela, M. (1995). Estados psicológicos, salud y enfermedad. Avances en Psicología Clínica Latinoamericana, 13, 1, 53-57.

Mustaca, A. E., Bentosela, M., Ruetti, E., Kamenetzky, G., Cuenya, L. et al. (2009). Similitudes y discrepancias en dos modelos animales de frustración. En M. C. Richaud de Minzi y Eduardo Moreno (Eds.) nombre del libro. (Tomo II, pp. 921-940). Ediciones CIIPME-CONICET

Mustaca, A., Freidín, E., \& Papini, M. (2002). Extinction of Consummatory Behavior in Rats. International Journal of Comparative Psycho$\log y, 15,1-10$.

Mustaca, A., \& Martínez, C. (2000). Respuestas agonísticas en ratas sometidas a frustración. Revista Latinoamericana de Psicología, 32, 485-504.

Mustaca, A. Martínez, C., \& Papini, M. R. (2000). Surprising nonreward reduces aggressive behavior in rats. International Journal of Comparative Psychology, 13(1), 91-100.

Nation, J. R., \& Cooney, J. B. (1982). The time course of extinction-induced aggressive behavior in humans: evidence for a stage model of extinction. Learning and Motivation, 13, 95-112.

O'Kelly, L. E., \& Steckle, G. C. (1939). A note on long-enduring emotional responses in the rat. Journal of Psychology, 8, 125-131.

Overmier, J. B., \& Seligman, M. E. P. (1967). Effect of inescapable shock upon subsequent escape and avoidance learning. Journal of Comparative and Physiological Psychology, 63, 23-33.

Papini, M. R. (2003). Comparative psychology of surprising nonreward. Brain, Behavior and Evolution, 62, 83-95.

Papini, M. R., \& Dudley, R. T. (1997). Consequences of surprising reward omissions. Review of General Psychology, 1, 175-197.
Papini, M. R., Fuchs, P. N., \& Torres, C. (2015). Behavioral neuroscience of psychological pain. Neuroscience \& Biobehavioral Reviews, 48, 53-69.

Pellegrini, S., Muzio, R. N., Mustaca, A. E., \& Papini, M. R. (2004). Successive negative contrast after partial reinforcement in the consummatory behavior of rats. Learning and Motivation, 35, 303-321.

Pérez Manrique, T., Díaz Barbosa, J. H., Pulido Vega, J. C.(2009). Contraste conductual en operantes relacionales con participantes humanos. Acta Colombiana de Psicología, 12(2), 115-126.

Priks, M. (2010). Does frustration lead to violence? Evidence from the Swedish hooligan scene. Kyklos, 63, 450-460.

Purgert, R. J., Wheeler, D. S., McDannald, M. A., \& Holland, P. C. (2012). Role of Amygdala Central Nucleus in Aversive Learning Produced by Shock or by Unexpected Omission of Food. The Journal of Neuroscience, 32(7), 2461-2472.

Rando, T. A. (1993). An investigation of grief and adaptation in parents whose children have died from cancer. In M. C. Roberts \& G. P. Koocher (Ed.), Readings in pediatric psychology, (pp. 127-144). New York: Plenum Press.

Rees, D., \& Schnepel, K. (2009). College football games and crime. Journal of Sports Economics, 10, 68-86.

Retana-Márquez, S., Bonilla-Jaime, H., \& Velázquez-Moctezuma. (1998). Lack of effect of corticosterone administration on male sexual behavior of rats. Physiology \& Behavior, 63, 367-370.

Retana-Márquez, S., Salazar, E. D., \& Velázquez-Moctezuma, J. (1996). Effect of acute and chronic stress on masculine sexual behavior in the rat. Psychoneuroendocrinology, 21, 39-50.

Richard, J., \& Knutson, J. F. (1983) Effects of reactivity to dorsal stimulation and social role on aggressive behavior in laboratory rats. Aggressive Behavior, 9, 287-301. 
Ruetti, E., Justel, N., Mustaca, A., Torrecilla, M., \& Gonzalez Jatúff, A. (2010). Estrés neonatal y frustración. Revista Latinoamericana de Psicología, 42, 2, 279-288.

Scully, J., A., Tosi, H., \& Banning, K. (2000). Life events checklists. Revisiting the social readjustment rating scale after 30 years. Educational and Psychological Measurement, 60, 864-876.

Sosa, M. T., Racki, M. Khoury, M.,Vvillarreal, R., Cestari,G., Melemt, E., \& Sosa Liprandi, A. S. (2012). Crisis económico-financieras en la Argentina: ¿un nuevo factor de riesgo de mortalidad cardiovascular? Revista Argentina de Cardiología, 80, 137-144.

Stein, M., \& Trestman, R. L. (1990). Anxiety and the immune system. In J. C. Ballenger (Ed.), Neurobiology of panic disorder (pp. 333-348). New York: Wiley-Liss.

Tinklepaugh, O. L. (1928). An experimental study of representative factors in monkeys. Journal of Comparative Psychology, 8, 197-236.

Tironi, E. (1989). ¿Pobreza=frustración=violencia? Crítica empírica a un mito recurrente. Working paper. Recuperado de https://kellogg.nd.edu/ publications/workingpapers/WPS/123.pdf

Twenge, J. M., Roy F. Baumeister, R. F., DeWall, C. N., Ciarocco, N. J., \& Bartels, M. (2007). Social Exclusion Decreases Prosocial Behavior. Journal of Personality and Social Psychology, 92, 1, 56-66.

Ulrich, R. E., \& Azrin, N. H. (1962). Reflexive fighting in response to aversive stimulation. Journal of the Experimental Analysis of Behavior, 5, 511-520.

Verona, E., Reed, A., Curtin, J. J., \& Pole, M. (2007). Gender differences in emotional and overt/co-

\section{Recibido: febrero 24, 2016 Aprobado: febrero 20, 2017}

vert aggressive responses to stress. Aggressive Behavior, 33, 261-271.

Vindas, M. A., Ole F., Kristiansen, T. S., Stien, L. H., Braastad, B., Mayer, I, \& Øverli, Ø. (2012). Omission of expected reward agitates Atlantic salmon (Sal.O.mo salar). Animal Cognition, 15, 903-911.

Wang, Y., \& Zhong, Z. (2015). Effects of frustration situation and resilience on implicit aggression. Chinese Journal of Clinical Psychology, 23(2), 209-212.

Wann, D. L., Culver, Z., Akanda, R., Daglar, M., De Divitiis, C., et al. (2005). The Effects of Team Identification and Game Outcome on Willingness to Consider Anonymous Acts of Hostile Aggression. Journal of Sport Behavior, 28, 282-294.

Williams, J. L. (1984). Influence of postpartum shock controllability on subsequent maternal behavior in rats. Animal Learning and Behavior, 12, 209-216.

Williams, J. L., \& Lierle, D. M. (1986). Effects of stress controllability, immunization and therapy on the subsequent defeat of colony intruders. Animal Learning and Behavior, 14(3), 305-314.

Williams, K. D., Cheung, C. K. T., \& Choi, W. (2000). Cyberostracism: Effects of being ignored over the internet. Journal of Personality and Social Psychology, 79, 748-762.

Yates, A. J. (1975). Frustración y conflicto. Ed. Josefina Betancor, Madrid, España.

Yoon, O. K., \& Joo, H. J. (2005). A contextual analysis of crime rates: The Korean case. Crime, Law, and Social Change, 43, 31-55.

Zadro, L., \& Gonsalkorale, K. (2014). Sources of Ostracism: The Nature and Consequences of Excluding and Ignoring Others. Current Directions in Psychological Science, 23(2), 93-97. 
